\title{
Das deutsche Klimaziel im europäischen Kontext: strategische Implikationen im Wahljahr 2013
}

\author{
Oliver Geden und Ralf Tils
}

\begin{abstract}
Kernaussagen
Schon seit 2007 steht das rein deklaratorische deutsche Klimaziel von $40 \%$ in einem latenten Widerspruch $\mathrm{zu}$ den rechtsverbindlichen Verpflichtungen gegenüber der EU, die sich bis 2020 lediglich auf etwa 33\% belaufen. Die Notwendigkeit einer Implementierung der 2. Verpflichtungsperiode des Kyoto-Protokolls in EU-Recht könnte diesen Widerspruch ausgerechnet im Wahljahr erstmals aufbrechen lassen. Die wahl- und koalitionsstrategischen Erwägungen der beiden Volksparteien lassen jedoch eher erwarten, dass die Klimaziel-Frage im Bundestagswahlkampf bewusst de-thematisiert wird.
\end{abstract}

\section{Nationales Vorreiterbewusstsein - europäische Regulierungshoheit}

Fragen des Umwelt- und Klimaschutzes genießen in Deutschland einen vergleichsweise hohen Stellenwert. Dies gilt, wenn auch mit starken Differenzen zwischen den soziokulturellen Milieus, nicht nur für die Bevölkerung (vgl. Rückert-John et al. 2013), sondern auch für Medien, Ministerialverwaltungen und Parteien. Insbesondere in der Klimapolitik nimmt sich Deutschland als internationaler Vorreiter wahr, und dies völlig zu Recht. Entgegen dem globalen Trend sind die Treibhausgasemissionen hierzulande seit 1990 um knapp 26\% gesunken, wenn auch stark begünstigt durch den Zusammenbruch der Industrie in Ostdeutschland. Über das Ziel, die deutschen Emissionen bis zum Jahr 2020 um 40\% zu senken, besteht zwischen allen (!) im Bundestag vertretenen Parteien breiter Konsens. Die Zielmarke liegt sämtlichen staatlichen Energiewendeszenarien zugrunde. In der Bevölkerung gilt der Klimaschutz als eines der Leitthemen der Energiewende (vgl. Bundesverband der Energie- und Wasserwirtschaft 2013).

Auch innerhalb der EU, die sich als globale Führungsmacht im Klimaschutz begreift, nimmt Deutschland eine Vorreiterposition sein. Die EU-27 hat ihre Emissionen seit 1990 zwar insgesamt um fast $18 \%$ gesenkt. Doch mehr als ein Drittel dieser Reduktion geht auf das Konto der größten europäischen Volkswirtschaft. Auch politisch zählt Deutschland traditionell zu den treibenden Kräften. So hat die Bundesregierung etwa die EU-Position bei den Verhandlungen zum Kyoto-Protokoll 1997 entscheidend geprägt, unter maßgeblicher Beteiligung der damaligen Bundesumweltministerin Angela Merkel. Auch die Tatsache, dass die EU seit 2007 die Minderung von Emissionen in den Mittelpunkt ihrer Energiestrategie stellt, ist ganz wesentlich auf den Einsatz der seinerzeit amtierenden deut- schen Ratspräsidentschaft zurückzuführen (vgl. Geden/ Fischer 2008).

Seit 2010 jedoch befindet sich die EU-Klimapolitik in einer anhaltend tiefen Krise, die auch die Bundesregierung vorsichtiger agieren lässt. Mit dem gescheiterten Klimagipfel von Kopenhagen Ende 2009 haben sich die Hoffnungen auf den baldigen Abschluss eines ambitionierten globalen Klimaabkommens zerschlagen. Die Finanz-, Wirtschafts- und Schuldenkrise hat die politischen Prioritätensetzungen in der EU zudem drastisch verschoben. Im energiepolitischen Zieldreieck aus Nachhaltigkeit, Versorgungssicherheit und Wettbewerbsfähigkeit wird der letztgenannte Aspekt inzwischen wieder sehr viel stärker betont, insbesondere im Hinblick auf das Energiepreisniveau, nicht zuletzt verglichen mit den USA. Der „wissenschaftsbasierte“ Ansatz einer nachhaltigkeitszentrierten Energiepolitik, der Emissionspfade bis ins Jahr 2050 skizziert und verbindliche Zwischenziele mit einem Vorlauf von mehr als zehn Jahren festzuschreiben versucht, wäre in absehbarer Zeit zwar ohnehin ins Wanken geraten, da er zum dominanten inkrementellen Modus des Politikbetriebs im Widerspruch steht. Die wirtschaftliche Krise in Europa beschleunigt diesen Prozess jedoch, da sie ein populäres Begründungsmuster für eine Kurskorrektur stärkt.

Paradoxerweise sind es vor allem die mit den Produktionsrückgängen in Europa einhergehenden, gewissermaßen „ungeplanten“ Emissionsminderungen, die die EU-Klimapolitik schon jetzt unter massiven Reformdruck geraten lassen. Da 2007 ein EU-Emissionsminderungsziel von 20\% bis 2020 vereinbart worden war, bis Ende 2011 aber bereits 17,6\% erreicht wurden, ist jeglicher klimapolitischer Elan erloschen. Die Preise im Emissionszertifikatehandel sind eingebrochen. Vom zentralen Instrument der EU-Klimapolitik geht keinerlei ökonomische Transformationswirkung mehr aus. Hinsichtlich möglicher Reformen in der EU-Klimapolitik verhält sich selbst die Bundesregierung ausgesprochen passiv, da das FDPgeführte Bundeswirtschaftsministerium (BMWi) die Beibehaltung des Status Quo favorisiert und den Reformeifer des CDUgeführten Umweltministeriums (BMU) regelmäßig auszubremsen vermag.

Diese Konflikte werden hierzulande jedoch nur von einer Fachöffentlichkeit wahrgenommen, da die EU-Klimapolitik im breiteren medienöffentlichen Diskurs nicht als eigenständige Handlungsebene identifiziert wird, die zudem auch einen starken Einfluss auf Deutschland haben könnte. In dem seit zwei Jahrzehnten dominanten klimapolitischen Deutungsmuster wird die EU primär als Anhängsel einer deutschen Vorreiterpolitik verortet, eine Sichtweise, die sich nach den Energiewende-Beschlüssen eher noch verstärkt hat. Wenn es 
Deutschland gelingt, die Transformation zu einer $\mathrm{CO}_{2}$-armen Volkswirtschaft erfolgreich zu bewältigen, werden die übrigen Europäer früher oder später folgen. Und so lange sie es nicht oder nicht im annähernd gleichen Tempo tun, wird Deutschland unbeirrt seinen Weg gehen. In die Sphäre klimapolitischer Ziele übersetzt sich dieses Deutungsmuster wie folgt: Ganz gleich, ob die EU ihre Emissionen bis 2020 um 20, 25 oder $30 \%$ senken will, Deutschland selbst ist davon nicht tangiert und verfolgt auf jeden Fall ein nationales 40\%-Ziel.

De facto sind die Verhältnisse jedoch weit komplexer, als es diese Selbstdeutung vermuten ließe. Anders als die Energiepolitik, in der das Letztentscheidungsrecht über den Energieträgermix nach wie vor bei den Mitgliedstaaten liegt, zählt die Klimapolitik in der EU zu den „vergemeinschafteten“ Politikfeldern. Zentrale Entscheidungen werden also nicht mehr auf nationaler Ebene getroffen, sondern in Brüssel, entweder als strategische Grundsatzbeschlüsse bei den Gipfeltreffen der Staats- und Regierungschefs oder im ordentlichen Gesetzgebungsverfahren zwischen Ministerrat und Europäischem Parlament. Übergreifende Emissionsreduktionsziele - wie etwa die $20 \%$ bis 2020 - werden zunächst von den Staats- und Regierungschefs beschlossen, und zwar einstimmig, also mit Vetorecht für jeden Mitgliedstaat. Die detaillierte rechtsverbindliche Umsetzung dieser Beschlüsse erfolgt erst im Anschluss. Bei der Aushandlung von EU-Rechtsakten tritt das Parlament (mit einfacher Mehrheit) als Ko-Gesetzgeber auf. Im Ministerrat bedarf es auch nicht mehr der Einstimmigkeit unter den Mitgliedstaaten, sondern lediglich einer qualifizierten Mehrheit. Theoretisch könnte Deutschland im Gesetzgebungsverfahren also überstimmt werden, in jedem Fall aber muss eine Bundesregierung dort Kompromisse eingehen.

Das EU-Klimaziel wird derzeit vor allem über zwei Instrumente regulatorisch umgesetzt, zum einen über das Emissionshandelssystem (ETS), an dem europaweit etwa 12.000 Kraftwerke und Industrieanlagen teilnehmen müssen, zum anderen mittels Emissionsobergrenzen für alle nicht vom ETS abgedeckten Sektoren (Verkehr, Gebäude, Landwirtschaft etc.). Während im Non-ETS-Bereich nach wie vor nationale Ziele existieren, deren Einhaltung die Mitgliedstaaten gewährleisten müssen, ist die Zuteilung der Zertifikate im Emissionshandel seit 2013 vollständig europäisiert.

Das deutsche Klimaziel von $40 \%$ bis 2020 ist vor dem Hintergrund des europäischen Rechtsrahmens deshalb primär als ein deklaratorisches zu verstehen. Die tatsächlichen rechtsverbindlichen Reduktionsverpflichtungen liegen deutlich darunter, seriöse Schätzungen gehen von 33\% aus (vgl. Expertenkommission 2012), abhängig von den zugrundegelegten Annahmen bewegen sie sich zwischen 31 und 35\%. Dass dies im deutschen klimapolitischen Diskurs bislang ausgeblendet werden konnte, ist primär auf den Umstand zurückzuführen, dass es die Europäisierung des ETS kalkulatorisch erschwert, die zu erbringenden Emissionsreduktionen noch einzelnen Mitgliedstaaten zuzuordnen. Dies wäre auch politisch nur begrenzt sinnvoll, da die Steuerungsmöglichkeiten der natio- nalen Regierungen bei EU-weit börsengehandelten Zertifikaten gegen Null tendieren.

\section{2 „Störfaktor“ Kyoto-II}

Der latente Widerspruch zwischen dem bloß deklaratorischen deutschen Klimaziel und den rechtsverbindlichen Verpflichtungen im EU-Kontext bricht im politischen Diskurs nur unter zwei Bedingungen nicht auf. Zum einen muss in Deutschland die Einschätzung vorherrschend bleiben, dass das 40\%-Ziel auch tatsächlich erreichbar ist. Zum anderen darf seitens der EU kein alternativer Zielwert formuliert werden, der die tatsächlichen deutschen Verpflichtungen umreißt und $\mathrm{zu}$ den bereits etablierten $40 \%$ in Konkurrenz tritt.

Keine Untersuchung geht gegenwärtig davon aus, dass Deutschland auf dem Weg sei, sein 40\%-Ziel bis 2020 auch tatsächlich zu erreichen. Auf Basis der bislang beschlossenen Maßnahmen gilt ein Korridor von 30-35\% als realistisch. Um die $40 \%$ noch zu schaffen, wäre eine Verdopplung der durchschnittlichen jährlichen Reduktionsraten notwendig (vgl. Matthes et al. 2013; Ziesing 2013). Die Einhaltung des 40\%Ziels würde also erhebliche zusätzliche Anstrengungen erfordern, ein keineswegs unmögliches, aber dennoch unwahrscheinliches Unterfangen, zumal die Emissionen 2012 gegenüber dem Vorjahr nach vorläufigen Schätzungen um 1,6\% gestiegen sind. Da es zudem ausgeschlossen scheint, dass sich die EU noch auf eine gemeinsame Verschärfung ihres Klimaziels für 2020 verständigen wird, müssten die über die $33 \%$ hinausgehenden nationalen Minderungsanstrengungen jenseits des vollständig europäisierten Emissionshandels erbracht werden, vor allem in den elektoral äußerst sensiblen Sektoren Verkehr und Gebäude.

In der Logik des Politikbetriebs wäre es dementsprechend naheliegend, dass eine (zumindest teilweise) erneuerte Regierungskoalition nach den Bundestagswahlen 2013 oder 2017 das 40\%-Ziel bis 2020 für unerreichbar erklärt, die Verantwortung dafür auf einer der Vorgängerregierungen ablädt und sogleich ein ehrgeiziges Klimaziel für 2030 beschließt. Es würde also erst im Moment der „Bereinigung“ thematisiert werden müssen, dass die tatsächlichen Verpflichtungen Deutschlands stets niedriger ausgefallen waren als die im breiten Konsens deklarierten nationalen Ziele - was erst den Raum dafür geöffnet hatte, die 2007 erstmals verkündete nationale Zielmarke nur halbherzig zu verfolgen. Würde Deutschland jedoch schon früher zu einer Differenzbereinigung gezwungen, müssten die politischen Akteure eine Neubewertung der Lage vornehmen. Sollte eine solche Situation noch vor der Bundestagswahl am 22. September 2013 eintreten, würde diese sehr stark von parteipolitischen Erwägungen geprägt sein. Der bislang stabile $40 \%$-Konsens könnte dann schnell aufbrechen.

Ausgangspunkt eines solchen Szenarios ist die beim Weltklimagipfel in Doha Ende 2012 beschlossene 2. Verpflichtungsperiode des Kyoto-Protokolls und deren Umsetzung in EU-Recht. Aufgrund der schleppend verlaufenen UN-Verhandlungen und dem mangelnden Ehrgeiz anderer Industrie- 
und Schwellenländer hatte sich die EU entschieden, auf UNEbene nur solche Minderungspflichten einzugehen, die sie sich selbst bereits auferlegt hat, also $20 \%$ bis 2020 . Wie schon der Beschluss zur 1. Verpflichtungsperiode enthält auch die Anschlussregelung lediglich ein Ziel für die gesamte EU, jedoch keinen Hinweis auf die konkreten Verpflichtungen ihrer Mitgliedstaaten. Ausgehend von Artikel 4 des KyotoProtokolls wurde bei der Implementierung von Kyoto-I jedoch in einer Entscheidung des Ministerrates eine rechtlich verbindliche Aufteilung des gesamteuropäischen Minderungsbeitrags auf die Mitgliedstaaten vorgenommen, ein Rechtsakt, auf den bei der Hinterlegung der mitgliedstaatlichen Ratifikationsurkunden bei der UN ausdrücklich verwiesen werden musste. Aufgrund des EU-internen „burden sharing“ betrug das rechtlich verbindliche Kyoto-I-Ziel Deutschlands 21\%, das EUGesamtziel lediglich 8\%.

Wählt die EU bei der Implementierung von Kyoto-II eine ähnliche Herangehensweise, so wird sie erstmals gezwungen sein, das seit 2007 bestehende EU-Klimaziel von $20 \%$ in einem ordentlichen Gesetzgebungsverfahren exakt auf mitgliedstaatliche Minderungsbeiträge herunter zu brechen. In der PolicyDimension von Klimapolitik mag diese Aufteilung aufgrund des gesamteuropäisch organisierten ETS methodisch fragwürdig erscheinen, zudem rechtssystematische Fragen aufwerfen, da eine neuerliche „burden sharing“-Entscheidung die im EURecht bereits bestehenden Verpflichtungen quasi „verdoppelt“. In der Politics-Dimension aber kreiert dieser Prozess unmittelbar neue Evidenzen. Schon der erste KommissionsEntwurf einer bislang inexistenten Tabelle nationaler Minderungspflichten bis 2020 wird nicht nur die Bundesregierung, sondern alle im Bundestag vertretenen Parteien nötigen, neu über ihre Positionierung zu entscheiden. Dabei geraten zwei Status Quo-Logiken miteinander in Konflikt: der faktisch europarechtliche, aber weithin unbekannte Status Quo einer Minderungsverpflichtung von 33\% und der lediglich politisch-deklaratorische Status Quo des nationalen 40\%-Ziels. Zwar wäre zu erwarten, dass sich die überwiegende Zahl der Akteure für eine Beibehaltung des 40\%-Ziels ausspricht. Da eine Verfehlung dieses Ziels unter den neuen rechtlichen Bedingungen jedoch mit signifikanten politischen und monetären Kosten verbunden wäre, würde das reale klimapolitische Ambitionsniveau in Deutschland ebenfalls steigen müssen, möglicherweise zusätzlich abgesichert durch ein nationales Klimaschutzgesetz.

\section{Strategische Implikationen einer deutschen Klimaziel- Debatte}

Bei der Entscheidung, welchen Minderungsbeitrag Deutschland im EU-Kontext zukünftig verpflichtend übernehmen will, sind drei Grundvarianten denkbar:

(1) Der deutsche Minderungsbeitrag liegt bei 33\%, das EUZiel bleibt bei $20 \%$.

(2) Der deutsche Minderungsbeitrag liegt bei $40 \%$, das EUZiel bleibt bei $20 \%$, die Verpflichtungen anderer Mitglied- staaten reduzieren sich, weil Deutschland seinen Beitrag faktisch erhöht.

(3) Der deutsche Minderungsbeitrag liegt bei $40 \%$, die Verpflichtungen der anderen Mitgliedstaaten bleiben gleich, das EU-Ziel erhöht sich (auf ca. 22\%), weil Deutschland seinen Beitrag faktisch erhöht.

Bei der Frage, wie sich Parteien, Ministerien und Stakeholder (etwa Wirtschafts- und Umweltverbände) zu diesen Optionen verhalten werden, ist nicht zuletzt der politische Kalender maßgeblich, nicht nur die Bundestags- und Landtagswahlen im September 2013, sondern vor allem auch die zeitliche Taktung der Prozesse auf EU-Ebene. Sollte es nicht zu Interventionen wichtiger Mitgliedstaaten kommen, die für eine Verschiebung des Prozesses plädieren, so müsste die Kommission spätestens im Sommer 2013 einen Vorschlag präsentieren, mit dem das EU-Gesetzgebungsverfahren beginnt. Da dieses jedoch erst 2014 zum Abschluss gebracht werden kann, ist die amtierende Bundesregierung nicht gezwungen, sich bereits vor der Wahl auf eine definitive Verhandlungsposition festzulegen. Sollte die Frage des deutschen Klimaziels zu einem Thema im Bundestagswahlkampf werden, könnten die dabei eingenommenen Positionen allerdings das Regierungshandeln während der kommenden Legislaturperiode vorstrukturieren, zumal die Bedeutung des Klimaschutz-Issues bis 2015 absehbar zunehmen wird. So wird der Weltklimarat IPCC schon in der Woche nach der Bundestagswahl Teil 1 seines 5. Sachstandsberichts (dem ersten seit 2007) präsentieren, die restlichen Teile dann sukzessive bis zum Herbst 2014. Ende 2015 schließlich soll auf UN-Ebene ein umfassender Weltklimavertrag beschlossen werden, kurz darauf vermutlich auch neue EU-Energie- und Klimaziele für die Zeit nach 2020 (vgl. Geden 2012 a).

Anders als für die EU-Institutionen und die nationalen Ministerialverwaltungen endet die Orientierungsperspektive der politischen Parteien und ministeriellen Leitungsebenen in Deutschland vorläufig am Tag der Bundestagswahl. Sofern die Frage „40 oder 33?“ schon im Sommer 2013 von der europäischen auf die nationale Ebene hinüber schwappt, wird die Symbolik der Zielwerte jegliche Erwägungen über das Verhältnis nationaler Klimaziele und europäischer Regulierungshoheit überlagern - und policy-rationale Modi der Entscheidungsfindung eher behindern. In der für den Klimadiskurs charakteristischen Gut/Böse-Unterscheidung würden die Rollen klar verteilt sein. Nur wer für die $40 \%$ eintritt, nimmt seine Verantwortung für das Weltklima wahr. Wer für 33\% plädiert, wird mit Etikettierungen à la „Klimakiller“ umzugehen haben, verbunden mit dem - politisch-deklaratorisch richtigen, europarechtlich aber falschen - Vorwurf, die 33\%-Befürworter wollten das deutsche Klimaziel abschwächen. Für explizite Anhänger des niedrigeren Zielwerts bleibt nur der Wechsel der diskursiven Arena und die Aktivierung zweier klassischer Deutungsmuster der Wirtschafts- und Europapolitik: Zum einen gefährde eine zu ehrgeizige Klimapolitik die deutsche Wettbewerbsfähigkeit, zum anderen sei es an der Zeit, dass Deutschland seine Verpflichtungen gegenüber der 
EU lediglich 1:1 umsetze statt Vorgaben aus Brüssel stets überzuerfüllen. In Kurzform: „Keine zusätzlichen Belastungen!“

Aus der Perspektive der an ehrgeizigen Klimaschutzvorgaben interessierten, aber nicht parteipolitisch gebundenen Akteure (Umweltverbände, BMU-Arbeitsebene, umweltpolitische Think Tanks und Beratungseinrichtungen) birgt die Thematisierung der Frage „40 oder 33?“ ein strategisches Dilemma. Wenn Deutschland mit den derzeit implementierten Maßnahmen nur auf eine Emissionsreduktion von 30-35\% zusteuert und dies europarechtlich auch nicht sanktioniert werden kann, dann birgt die Herbeiführung einer Entscheidungssituation in ihrem sachlogischen Kern keinerlei Verlustrisiken, schließlich würden die rechtlichen Emissionsvorgaben selbst im schlechtesten Fall auf dem gegenwärtigen Niveau bleiben. Dennoch ist die Angst vor einer expliziten Festschreibung eines 33\%-Ziels beträchtlich, dementsprechend groß ist die Zurückhaltung in der deutschen klimapolitischen Community. Hierfür lassen sich vor allem drei Gründe anführen. Erstens der symbolische Wert möglichst anspruchsvoller Klimaziele, der unabhängig vom real bislang Geleistetem funktioniert (vgl. Geden 2012 b) und international ein VorreiterImage Deutschlands stützt, das in der alltäglichen Praxis des globalisierten Klimapolitikbetriebs auch positiv auf jeden deutschen Vertreter abstrahlt. Zweitens die (bislang ungedeckte) Hoffnung, dass die Existenz eines rechtlich unverbindlichen $40 \%$-Ziels bewirkt, dass Deutschland (freiwillig) mehr tun wird als zur Erfüllung der EU-Vorgaben notwendig ist. Und drittens schließlich würde eine offizielle Absenkung des deutschen Klimaziels auf 33\% auch die diskursiven Spielräume der Klimacommunity einschränken, denn der hier vorherrschende Kritikmodus zielt meist auf die Differenz zwischen regierungsamtlich formulierten Ansprüchen und der oft wesentlich bescheideneren Wirklichkeit, sprich: Je höher das deklarierte deutsche Klimaziel, desto mehr Raum für blaming and shaming.

Sollte die EU-Kommission ihren Vorschlag zur europarechtlichen Umsetzung von Kyoto-II noch vor der Bundestagswahl präsentieren und dabei jedem Mitgliedstaat ein (vorläufiges) nationales Minderungsziel zuweisen, werden die Parteien die „40 oder 33“-Frage erstmals zu berücksichtigen haben. Doch anders als in der fachpolitischen Community angenommen, sprechen die Besonderheiten eines Wahljahrs eher gegen eine (Vor-)Entscheidung. Die wahl- und koalitionsstrategischen Erwägungen der beiden Volksparteien lassen Versuche einer De-Thematisierung der Klimaziel-Frage erwarten. An einer offensiven Thematisierung sind lediglich die Grünen und möglicherweise auch die FDP interessiert.

Auf Seiten der Regierungskoalition spricht aus Sicht der Unionsparteien alles dafür, eine entsprechende Debatte vor der Bundestagswahl möglichst klein zu halten - und zwar aus drei strategischen Gründen. Erstens benötigen CDU/CSU ehrgeizige Klimaziele weder zur politischen Profilierung noch zur Mobilisierung ihres eigenen Wählerpotentials. Die Konzentration liegt in diesem Wahlkampf auf der Popularität der Kanzlerin, der ökonomischen Kernkompetenz der Union sowie einer sozialpolitischen Flankierung, die vor allem der SPD den Wind aus den Segeln zu nehmen versucht. Damit diese bereits im Wahlkampf 2009 erprobte Strategie der „asymmetrischen Demobilisierung“ aufgeht, müssen CDU/ CSU aber auch den Eindruck eines Abrückens vom 40\%-Ziel vermeiden. Nicht nur, weil dieses Ziel erstmals in der Großen Koalition unter Führung von Angela Merkel beschlossen wurde, sondern auch, weil dessen Abschwächung durch die Opposition zur Wählermobilisierung genutzt werden könnte. Zweitens liegt eine De-Thematisierung aus Sicht der Union auch deshalb nahe, weil anderenfalls latente Konflikte innerhalb des Regierungsbündnisses virulent werden und das Bild einer zerstrittenen Koalition abermals aktualisieren könnten. Nimmt man die bisher schon restriktiven Positionen der FDP in der europäischen Klimapolitik (keine Erhöhung des EUKlimaziels für 2020 und keine Reform des ETS) sowie bei der Förderung der Erneuerbaren zum Maßstab, so könnten die Liberalen auch bei einer Debatte über das deutsche Klimaziel geneigt sein, ihr wirtschaftspolitisches Alleinstellungsmerkmal in doppelter Abgrenzung von den Grünen und CDU/CSU hervorzuheben, indem sie offensiv für ein 33\%-Ziel plädieren. Drittens wird die Union gerade wegen ihrer starken Position auf dem Wählermarkt frühzeitige Festlegungen bei potenziell strittigen Fragen vermeiden wollen, um keine der für sie zahlreichen Koalitionsoptionen leichtfertig zu verkomplizieren: Eine aus heutiger Sicht wahrscheinliche Große Koalition hält andere klimapolitische Konfliktlinien bereit als eine Fortsetzung von Schwarz-Gelb oder gar ein schwarz-grünes Experiment.

Die Möglichkeiten eines aktiven Agenda-Cutting sind für die Union mannigfaltig, denn die CDU stellt sowohl die Regierungschefin als auch die Spitze des in Klimaschutzfragen federführenden BMU. Zudem ist der Einfluss der Regierung des größten EU-Mitgliedstaats auf das Handeln der Kommission gerade in der Klimapolitik nicht zu unterschätzen. Da die „40 oder 33? “-Frage in Deutschland nur dann virulent wird, wenn die Kommission einen Gesetzgebungsvorschlag präsentiert, der eine entsprechende deutsche Positionierung erfordert, liegt es nahe, zunächst auf den Zeitplan Einfluss zu nehmen, etwa durch Erteilung von Prüfaufträgen durch den Ministerrat, was aufgrund der inhaltlichen und rechtssystemtischen Komplexität der Kyoto-II-Implementierung durchaus gerechtfertigt sein kann (vgl. European Climate Policy Group 2013). Auf diese Weise ließen sich möglicherweise auch Wege finden, die europarechtlichen Verpflichtungen der Mitgliedstaaten nicht in jeweils einer einzigen Zahl zu aggregieren, die dann mit national deklarierten Zielen in Konflikt geriete - eine Problematik, die auch Dänemark und Großbritannien betrifft. Hinzu kommt die Möglichkeit einer Profilierung von Problemlagen im gleichen thematischen Segment, vor allem hinsichtlich der Energiewende, etwa in Bezug auf eine Reform der Förderung der erneuerbaren Energieträger oder Fragen des Netzausbaus.

Die Opposition wirkt in der Klimazielfrage auf den ersten Blick relativ „geschlossen“. Linkspartei, Grüne und SPD würden wohl unisono für ein $40 \%$-Ziel plädieren, insbesondere 
falls die Bundesregierung zögerlich agieren oder die FDP gar offensiv für $33 \%$ eintreten würde. Deutliche Unterschiede wären bei der relativen Gewichtung im Wahlkampf zu erwarten. Während Klimapolitik im Themenportfolio der Linkspartei nur randständig ist, wäre eine Klimaziel-Debatte für die Grünen von beträchtlicher strategischer Relevanz. Zum einen erreichen die Grünen bei Umweltthemen nach wie vor die größten Kompetenzwerte aller Parteien. Zum anderen ließen sich in einer kontroversen Debatte auch deutliche Unterschiede zu FDP und Unionsparteien markieren, wie sie in der Energiepolitik nach dem Atomausstiegsbeschluss sukzessive verlorengegangen sind (vgl. Raschke/Tils 2012). Allerdings wirken hier die begrenzten eigenständigen Thematisierungspotentiale von Kleinparteien restriktiv. Den Grünen wird es selbst mit Unterstützung der NGOs kaum gelingen können, das Klimaschutzthema auf der bundespolitischen Agenda gegen konkurrierende wahlkampfprägende Issues durchzusetzen.

Der Umgang der SPD mit Klimaschutzfragen wäre im Bundestagswahlkampf sicherlich von einiger Ambivalenz gekennzeichnet. Sie würden das $40 \%$-Ziel schon deshalb nicht in Frage stellen, weil es 2007 vom damaligen Bundesumweltminister Sigmar Gabriel mitentwickelt wurde. Andererseits steht für die Sozialdemokraten im Wahlkampf 2013 vor allem die ökonomische und soziale Profilierung im Vordergrund, quer über alle Themenfelder hinweg. Eine starke Fokussierung der Wahlkampfauseinandersetzungen auf ein Klimathema würde das Bestreben der SPD unterlaufen, sich in der Energiepolitik - in Abgrenzung zu den Grünen - weniger auf den Aspekt der ökologischen Nachhaltigkeit zu konzentrieren, als auf soziale und wirtschaftspolitische Fragen, insbesondere auf Energiepreise für Privathaushalte und Industrie.

\section{Fazit}

Das Beispiel des deutschen Klimaziels im EU-Kontext zeigt anschaulich, wie Ansprüche nationaler Deutungshoheit im latenten Widerspruch zu einer faktischen europäischen Regulierungshoheit stehen können. Zwar mag die Auflösung eines solchen Widerspruchs durch die dynamische Eigenlogik des Policy-Felds befördert werden, im konkreten Fall durch die Notwendigkeit einer Umsetzung der Kyoto-II-Verpflichtungen in EU-Recht. Ob und in welchem Maße eine „Differenzbereinigung “ im deutschen politischen System vorgenommen wird, ist jedoch wesentlich von den Politics-Interessen der zentralen politischen Akteure abhängig. Policies sind ohne Politics nicht denkbar, Gestaltungsziele werden nicht unabhängig von Machtzielen verfolgt - auch nicht im Bereich vorsorgeorientierter Politikfelder (vgl. Tils 2005). Der kurze Blick auf die gegenwärtigen parteipolitischen Konstellationen und strategischen Priorisierungen zeigt, dass vor der Bundestagswahl 2013 mit einer umfassenden Politisierung der deutschen Klimaschutzziele nicht zu rechnen ist, weil insbesondere CDU/ CSU, aber auch die SPD nicht an einer frühzeitigen Positionierung interessiert sein werden. Zudem besetzen originäre Fragen des innenpolitisch weitaus wichtigeren Energiewende-
Issues in der Wahlkampfauseinandersetzung das gleiche thematische Segment.

$\mathrm{Ob}$ und in welcher Weise deklaratorisches und rechtsverbindliches Klimaziel in der kommenden Legislaturperiode miteinander in Übereinstimmung gebracht werden, hängt deshalb wesentlich von der Koalitionsbildung im Herbst 2013 ab. Bei der Beantwortung der Frage, ob Deutschland eine Reduktion um $40 \%$ bis 2020 tatsächlich ernsthaft verfolgen will, wird dann jedoch nicht mehr der Symbolwert eines möglichst ehrgeizigen Ziels im Vordergrund stehen. Eine komplette Legislaturperiode vor Augen, werden die Erwägungen der jeweiligen Regierungsparteien vielmehr stark davon geprägt sein, die konkreten Auswirkungen zielkonformer Maßnahmenkataloge zu antizipieren - und zwar nicht nur auf dem Energie-, sondern mindestens ebenso sehr auch auf dem Wählermarkt.

\section{Literatur}

Bundesverband der Energie- und Wasserwirtschaft 2013. BDEW Energiemonitor 2013: Das Meinungsbild der Bevölkerung. Berlin.

European Climate Policy Group (2013). Ratification of the Kyoto Protocol's 2nd commitment period, Background Document, 19 February 2013.

Expertenkommission zum Monitoring-Prozess "Energie der Zukunft " (2012). Stellungnahme zum ersten Monitoring-Bericht der Bundesregierung für das Berichtsjahr 2011. Berlin u.a.

Geden, O. (2012 a). Vor dem Paradigmenwechsel. Die internationalen Klimaverhandlungen und ihr Einfluss auf die Energiepolitik der EU, in: KAS Auslandsinformationen 9/2012, S. 24-36

Geden, O. (2012 b). Die Modifikation des 2-Grad-Ziels. Klimapolitische Zielmarken im Spannungsfeld von wissenschaftlicher Beratung, politischen Präferenzen und ansteigenden Emissionen. Stiftung Wissenschaft und Politik, Studie 12/2012. Berlin.

Geden, O. \& S. Fischer (2008). Die Energie- und Klimapolitik der Europäischen Union. Bestandsaufnahme und Perspektiven. Baden-Baden.

Matthes, F. et al. (2013). Politikszenarien für den Klimaschutz VI. Treibhausgas-Emissionsszenarien bis zum Jahr 2030. Dessau

Raschke, J. \& R. Tils (2012). Die Rationalität des grünen Traditionalismus oder: Die Suche nach Alternativen, in: Zeitschrift für Staats- und Europawissenschaften 4/2012, S. 510-539.

Rückert-John, J. et al. (2013). Umweltbewusstsein in Deutschland 2012. Ergebnisse einer repräsentativen Bevölkerungsumfrage. Berlin, Marburg.

Tils, R. (2005). Politische Strategieanalyse. Konzeptionelle Grundlagen und Anwendung in der Umwelt- und Nachhaltigkeitspolitik. Wiesbaden.

Ziesing, H.-J. (2013). Kühle Witterung und verstärkte Kohleverstromung lassen CO2-Emissionen in Deutschland 2012 steigen, in: Energiewirtschaftliche Tagesfragen 63 (5), S. 65-73.

Dr. Oliver Geden ist Senior Associate bei der Stiftung Wissenschaft und Politik (SWP). Nach seiner Promotion an der Humboldt- Universität zu Berlin war er u.a. an der UC Berkeley und im Planungsstab des Auswärtigen Amts tätig. Sein Forschungsschwerpunkt ist die Energie- und Klimapolitik der EU. E-Mail: oliver.geden@swp-berlin.org

PD Dr. Ralf Tils lehrt Politikwissenschaft an den Universitäten Bremen und Lüneburg. Er arbeitet in der Agentur für Politische Strategie (APOS). Seine Forschungs- und Kompetenzschwerpunkte liegen in den Bereichen politische Strategie, Parteien, Regierung und Verwaltung. E-Mail: tils@politischestrategie.de 